\title{
REAVI
}

\section{A ESCOLHA DE UMA INSTITUIÇÃO DE ENSINO SUPERIOR POR ESTUDANTES DO ENSINO MÉDIO}

\author{
Fabrício Sabino Carvalho $^{1}$, Caissa Veloso e Sousa ${ }^{2}$, Nayara Kelly Batista ${ }^{3}$ \\ Centro Universitário Unihorizontes ${ }^{1,2,3}$ \\ fabricio.carvalho@unihorizontes.br, caissaveloso@yahoo.com.br, \\ nayara.batista@unihorizontes.br
}

\begin{abstract}
Resumo
Este estudo objetivou analisar como os estudantes estabelecem suas decisões para a escolha de uma instituição de Ensino Superior, na perspectiva de alunos em curso do terceiro ano do Ensino Médio. Foram entrevistados 26 estudantes, a partir de um roteiro previamente elaborado, contendo as seguintes dimensões: grupos de referência, marca, programas de incentivo do governo, infraestrutura e preço. Os grupos de referência se mostraram a principal influência recebida dos estudantes na escolha da IES, sendo que parece haver um consenso sobre a formação da percepção à cerca da marca, a partir de compartilhamento de experiências com parentes e amigos próximos. Ainda, o artigo abre espaço para o questionamento à cerca do que seria a qualidade de ensino para os estudantes, considerando-se a intangibilidade do serviço e a dificuldade para o público discente avaliar aspectos pertinentes ao que seria uma estrutura que oferta serviços de qualidade. Destacase, como implicação gerencial, a importância de as IES formarem uma identidade da marca, que pode ser reconhecida por toda a comunidade de interesse.
\end{abstract}

Palavras-chave: Ensino Médio. Escolha da Escola. Marketing Educacional.

\section{CHOOSING A HIGHER EDUCATION INSTITUTION BY HIGH SCHOOL STUDENTS}

\begin{abstract}
The present study aims to analyze how third year high school students make up their mind in favor of a given higher education institution. 26 students were interviewed, on a previously prepared script containing the following dimensions: reference groups, brand, government incentive programs, infrastructure and price. Reference groups showed to be the main influence received from students in their choice of IES there seems to be consensus that perception of the brand arises chiefly from experiences shared with relatives and close friends. At this point, a consensus seems to close in upon the 'quality of the institution', molded by word of mouth. Moreover, the article makes room for questioning about what students would regard as quality of education, considering the intangibility of this service and the difficulty for the student public to assess relevant aspects of what would be a structure that offers quality services. Paramount as a managerial implication is that the HEIs form a brand identity that can be recognized by the community of interest.
\end{abstract}

Keywords: High school. School Choice. Educational Marketing.

Revista Eletrônica do Alto Vale do Itajaí - REAVI, v.08, n 13, p. 001-012, dez 2019 ISSN: 23164190, DOI 10.5965/2316419008112019001 


\section{REAVI}

\section{Introdução}

Tendo como referência a abertura econômica do país, inclusão de políticas públicas para a Educação Superior, identifica-se um aumento no número de Instituições de Ensino Superior (IES) no país. Tal cenário, para Cerchiaro e Mota (2010), faz com que os alunos aumentem as exigências na escolha por uma instituição de ensino superior.

Considerando esse contexto, se por um lado as instituições de ensino vivenciam um ambiente de concorrência acirrada (CERCHIARO; MOTA, 2010), no qual os estudantes são entendidos como consumidores de um serviço (PALÁCIO; MENESES; PÉREZ, 2002), por outro lado essas compreendem veículos que devem ofertar um serviço de qualidade, que atenda tanto aos anseios da promoção do ensino, quanto dos próprios alunos.

Não obstante, a educação, assim como todo serviço, é de difícil avaliação por seu usuário, dada algumas características, como: a falta de elementos físicos para sua avaliação, a produção e consumo simultâneos e a dificuldade de se oferecer uma padronização (ZEITHAML; BITNER, 2000; HOFFMAN; BATESON, 2006; LOVELOCK; WIRTZ, 2006).

A Educação Superior deve oferecer a todo o indivíduo uma participação essencial como cidadão, uma formação de competências para o processo educativo, inclusive os meios para avançar no trabalho e nos estudos posteriores. Nessa perspectiva, Gadotti (1997) afirma que as Organizações de Ensino Superior devem cumprir sua função social, contribuindo por meio da pesquisa e da extensão, para a solução dos problemas sociais, econômicos e políticos, nacionais e regionais, difundindo suas conquistas e resultados.

Neste aspecto, o prévio desenvolvimento dessas Instituições, em harmonia com as mudanças comportamentais dos alunos, criou possibilidades e ofertas de serviços para estimular e dar importância à formação profissional do aluno (SOUSA; NETO; FONTENELE, 2013).

Entretanto, Lbdaiwi (2009) descreve que, para sobreviver no ambiente prevalecente, especialmente nas primeiras décadas do século XX, de concorrência acentuada do mercado educacional, é importante o envolvimento e o conhecimento sobre como o aluno opta por uma instituição e quais são as concretizações consideradas no momento em que resolve obter uma formação em nível superior.

Segundo Alfinito (2002), a mensuração da qualidade nas IES é baseada em indicadores determinados pelos setores governamentais de controle de ensino e regulada por processos e parâmetros de avaliações sustentadas. Neste pensamento, o histórico da instituição influencia a decisão do estudante.

A partir do cenário apresentado, pretende-se analisar como estes estudantes estabelecem suas decisões para a escolha de uma Instituição de Ensino Superior, na perspectiva de alunos em curso do terceiro ano do Ensino Médio, na cidade de Belo Horizonte/MG.

\section{O Marketing para instituições educacionais}

Segundo Kotler e Fox (1994) e Manea e Purcaru (2017), o marketing para instituições educacionais é uma estratégia importante para que os objetivos dessas organizações sejam atingidos. Muitas IES o utilizam no momento em que a necessidade se faz presente, ou seja, quando se identifica que atrair novos alunos se torna mais difícil em função da concorrência no setor. O declínio no volume das matrículas pode ser um fator gerador dessa percepção.

Para Solomon (2002), essa imagem pública é importante por refletir nos grupos de referência que influenciam no comportamento, nas avaliações e nos anseios dos indivíduos. Ainda Revista Eletrônica do Alto Vale do Itajaí - REAVI, v.08, n 13, p. 001-012, dez 2019 ISSN: 23164190, DOI 10.5965/2316419008112019001 


\section{REAVI}

para o autor, entre esses grupos que desempenham papel central na formação dos valores, em relação a muitas questões importantes, estão: os pais e o núcleo familiar; podendo esses influenciarem na escolha de qual universidade frequentar.

Segundo Aléssio, Domingues e Scarpin (2010), a maioria dos estudantes procuram IES e cursos que possibilitem uma maior perceptibilidade e destaque profissional, independente da classe socioeconômica a qual pertencem. Para dar continuidade aos seus estudos, logo após o término do Ensino Médio, se amparam nos saberes de seus familiares para a escolha da área a ser cursada.

Especificamente sobre as IES, Pereira e Gil (2007) argumentam que é necessário empreender e empenhar esforços na captação de alunos. A utilização de recursos financeiros e ferramentas de comunicação podem ser consideradas na criação de vínculos com seu público-alvo, com o intuito de aumentar a satisfação dos alunos. Isso refletirá positivamente na percepção que as pessoas têm acerca da IES.

Michael (1997), afirma que o Ensino Superior trabalha em um mercado imperfeito, uma vez que os consumidores não têm conhecimento das condições de mercado prevalecentes. Por outro lado, conforme Koc (2006), o mercado de Ensino Superior, especialmente no início do século $\mathrm{XXI}$, tem forçado as IES privadas a encontrarem e adotarem estratégias de marketing mais práticas e inovadoras.

Assim, a imagem da IES está condicionada à percepção da marca e a outros fatores, como: prestígio, atendimento pré-matrícula, reconhecimento pelo Ministério da Educação e Cultura (MEC), tempo duração do curso, titulação do corpo docente, estrutura física, oportunidades de estágio, atividades extracurriculares, recursos tecnológicos, inovação, orientação de carreiras, responsabilidade social e orientação para a sustentabilidade. Estes são aspectos fundamentais que determinam o comportamento do consumidor e relação a escolha dos serviços educacionais (SOUSA, NETO, FONTENELE, 2013; KAGAWA, REICHELT, 2016).

Neste contexto, Siqueira e Carvalho (2006) criticam o direcionamento mercadológico dado às IES, uma vez que, para os autores, os alunos não devem ser puramente classificados como cliente da escola. Eles são os principais usuários do serviço educacional e são favorecidos diretamente pela qualidade da IES, que se adapta aos desígnios do mercado. Para o amadurecimento acadêmico dos alunos é importante incluir também os discentes, para a discussão a respeito da melhoria da oferta da prestação de serviço e para o aprimoramento de gestores, coordenadores e professores.

\section{Aspectos influenciadores da escolha de uma instituição de Ensino Superior}

As estratégias de marketing nas organizações devem ser inteligentes, eficazes e direcionadas, conforme o comportamento do cliente em adquirir produtos e serviços. Como a decisão de compra é um momento importante, saber analisar o que influencia nos processos de compra, como pensam e como agem o consumidor final é um diferencial para a organização (IBDAIWI, 2009).

Do ponto de vista de Seeman e O’Hara (2006), os alunos são atraídos por uma grande variedade de escolhas para pleitear o Ensino Superior. De fato, eles têm como opção o ensino a distância, as instituições de tecnologia e universidades tradicionais. Vários indicadores de eficiência são averiguados como fator principal de seleção pelos estudantes, como: as estatísticas de aprovação em exames do governo, a reputação acadêmica na sociedade e a taxa de diplomados ativos no mercado de trabalho.

Revista Eletrônica do Alto Vale do Itajaí - REAVI, v.08, n 13, p. 001-012, dez 2019 ISSN: 23164190, DOI 10.5965/2316419008112019001 


\section{REAVI}

Este cenário tem levado as IES a implantarem diversas estratégias para melhorar seu desempenho e atrair os clientes que estão cada vez mais exigentes (TARI, 2006). Para Foskett, Roberts e Maringe (2006), por exemplo, os estudantes com menor poder aquisitivo consideram os fatores econômicos como prioridade em tempos de dificuldades financeiras para a escolha de uma IES, priorizando o preço em questão.

Outra forma de atrair estudantes, segundo Mavondo, Chimhanzi e Stewart (2005), é inovar para melhorar o desempenho e solidificar no mercado. Para os autores, novos serviços e produtos, processos inovadores e articulação de ideias são quesitos básicos para um direcionamento vantajoso no mercado.

Para Martins et al. (2009), o crescimento concorrencial no mercado da educação atordoam os estudantes pela diversidade de instituições de ensino, o que cria a necessidade destas organizações identificarem os segmentos de mercado de seu interesse, as características e as necessidades dos seus clientes em potencial e a maneira como poderão atendê-los. Diante disso, é necessário posicionar o seu serviço e observar as ações dos concorrentes.

Segundo Almada e Tontini (2012), para escolher uma IES é necessário o entendimento de um difícil sistema implantado por propriedades intangíveis e fatores diversos. Nesse aspecto, podem ocorrer diferentes compreensões diante de atributos acordados entre consumidor e organização, bem como a presença de tendências cognitivas em decidir em qual instituição contratar (SANTOS et al., 2010).

À medida que o indivíduo obtém informações dos cursos em oferta e das profissões de seu interesse, aumenta a confiabilidade para decidir a escolha de um curso de graduação. Os estudantes são influenciados por fontes de opiniões e referências das pessoas com quem eles convivem. São vínculos favoráveis, apesar de receber deles informações incompletas e pouco esclarecidas, que acabam por gerar conhecimentos parciais e sem estruturação. A publicidade da IES também os orienta com conteúdos restritos e sem muito aprofundamento (LEVENFUS, NUNES, 2002).

Formas importantes de influências podem ser elencadas, como, por exemplo, os grupos de referência, que são aqueles grupos ou indivíduos que exercem influência significativa no comportamento do indivíduo, seja na intenção de se associar a ele ou dissociar-se dele (ENGEL, BLACKWELL, MINIARD, 2000); a marca institucional; os programas de incentivo do Governo disponíveis na IES (VIGGIANO; MATOS, 2013); a infraestrutura da IES (FROEMMING, 2001; MARTINS et al., 2009; DUDZIAK, 2010); e o preço das mensalidades (SOUSA; SOUSA, 2009).

\section{Metodologia}

Foi realizada uma pesquisa descritiva, de abordagem qualitativa. Os dados foram coletados por meio de entrevistas individuais, realizadas a partir de um roteiro semiestruturado. Foram entrevistados 26 estudantes em curso do Ensino Médio, alunos de escolas públicas e privadas. As entrevistas tiveram duração média superior a 20 minutos, sendo gravadas com a autorização dos entrevistados e, depois, transcritas. Todos os estudantes entrevistados moram e estudam na cidade de Belo Horizonte/MG. Para a elaboração do roteiro de entrevista foram propostas dimensões iniciais, elaboradas a partir da literatura que embasou o estudo.

Ressalta-se que as argumentações não ficaram restritas ao roteiro, sendo possível aos pesquisadores se aprofundarem em temáticas emergentes ao longo das entrevistas. Para a análise dos dados foram consideradas as proposições de Bardin (2006), no que se refere à análise categorial. Neste sentido, os dados coletados foram agrupados segundo características comuns

Revista Eletrônica do Alto Vale do Itajaí - REAVI, v.08, n 13, p. 001-012, dez 2019 ISSN: 23164190, DOI 10.5965/2316419008112019001 
encontradas nas falas dos entrevistados, a partir de categorias e subcategorias de análise definidas a priori e a posteriori.

\section{Apresentação e análise de resultados}

Inicialmente, procurou-se identificar o motivo declarado sobre o qual os estudantes fariam um curso superior. Nesse aspecto, treze entrevistados referiram-se à importância de se alcançar mais oportunidades no mercado de trabalho.

Sim, eu acho 'super' importante para o mercado de trabalho, e também na minha opinião para se dar bem na vida. (E5)

Muito importante, pelo fato de que hoje se a gente não tem um curso superior (...) a gente não consegue ingressar no mercado. (E6)

O significado de um curso superior, segundo alguns entrevistados, também pode representar a possibilidade de se diferenciar de uma condição de vida atual, conforme observado no relato E23.

Sim, porque hoje as pessoas que têm oportunidade de estar fazendo um curso superior, têm mais chance de estar ingressando no mercado de trabalho, e, com isso, mais chance de crescer na vida. E a minha intenção na vida e ser diferente da minha família, porque nela há pouquíssimas pessoas que tiveram oportunidade de ingressar na faculdade. E eu quero poder fazer a diferença. (E23)

Segundo Gadotti (1997), o Ensino Superior deve oferecer a todo indivíduo uma participação essencial como cidadão, uma formação de competências para o processo educativo, inclusive os meios para avançar no mercado de trabalho e nos estudos posteriores.

Quando questionados sobre as possíveis influências recebidas na escolha da IES, os entrevistados relataram, em sua maioria, a influência de grupos de referência primários, como a família e os amigos próximos, concordando com o que descreve Engel, Blackwell e Miniard (2000).

Já cheguei a pedir informação para amigos, familiares, para a escolha das faculdades. (E20)

Para solicitar informações, especificamente, eu perguntei ao meu irmão sobre o que ele pesquisou e o que ele acha. E ele falou quais são as mais em conta. (E26)

Neste sentido os alunos foram questionados sobre em qual IES têm preferência dar continuidade aos estudos. As Universidades Federais foram citadas por 16 entrevistados, conforme os relatos E12 e E13.

(...) eu já sei a instituição em que quero cursar. E a minha preferência é pelas Universidades Federais. (E12)

Eu ouço falar bem da UFMG, né, cara. Eu pretendo estudar na Federal (...) (E13)

Neste aspecto, reporta-se aos achados de Sousa e Sousa (2009), que também identificaram uma preferência pelas Universidades Federais, que parece estar mais associada a uma tradição do Revista Eletrônica do Alto Vale do Itajaí - REAVI, v.08, n 13, p. 001-012, dez 2019 ISSN: 23164190, DOI 10.5965/2316419008112019001 


\section{REAVI}

que a um conhecimento específico sobre os cursos ofertados. Para dois entrevistados, a Universidade Pública tem uma imagem mais positiva no olhar da sociedade.

Como segunda opção, onze entrevistados citaram outras IES localizadas na região metropolitana de Belo Horizonte, contudo, ressalta-se que estas seriam apenas uma 'segunda opção’.

Ainda sobre a marca, foi perguntado para os entrevistados o motivo da escolha dessa segunda marca e, novamente, se identifica a presença dos grupos de referência na formulação das escolhas individuais.

Porque meu irmão estudava nessa faculdade, e minha irmã, também. Então, minha Mãe indicou. (E2)

Ela vem crescendo muito, tá nova no mercado, tipo assim, estourou. Meu irmão faz faculdade lá. E só fala bem de lá. Nunca falou mal. Então, eu me interessei em fazer lá. (E3)

Porque ela é renomada e eu conheço várias pessoas que estudam lá e me deram uma boa indicação da faculdade, porque ela é referência em Minas Gerais e no mundo inteiro. (E22)

Ainda em conformidade com os achados de Sousa e Sousa (2009), os estudantes também citam a qualidade do ensino como fator importante para a escolha, mesmo que esses não consigam, por antecedência, afirmar o que seja essa qualidade no ensino. Quando questionados sobre esse aspecto, eles afirmaram que os cursos são reconhecidos como excelentes pela sociedade, as cargas horárias são produtivas e, ainda, destaca-se a formação dos professores.

Doze dos entrevistados relataram que o ensino é o ponto mais importante observado nas IES, conforme E2, E17 e E19.

Acho que é o ensino; o povo fala isso. (E2)

(...) porque os professores de lá cobram mais do aluno. Eles fazem ele ficar mais comprometido com a matéria. (E17)

Os procedimentos usados para ensinar os alunos. (E19)

Segundo Cardador e Pratt (2006), as instituições devem buscar o reconhecimento de particularidades que diferenciam um organização da outra. A qualidade e a metodologia de ensino são considerados diferenciais para os estudantes entrevistados.

Chama a atenção, neste aspecto, a forma como os entrevistados percebem os aspectos que tangenciam a qualidade do ensino, mesmo que para tanto não tenham experiências com os serviços prestados pela IES, vivenciando-as por meio de terceiros em quem confiam, o que reafirma a importância dos grupos de referência, como pais, amigos e, mais recentemente, as mídias sociais. Estas últimas têm-se mostrado importantes veículos para a busca de informações, principalmente para a geração de jovens do início do século XXI.

Dez entrevistados relataram buscar informações sobre as marcas de instituições de ensino superiores na internet, ressaltando a importância dessa forma de comunicação com o público em questão. Além da internet foram citados fóruns educativos e canais de TV aberta.

Apesar de a preferência relatada ser por uma IES pública, especialmente em função da imagem que essas transmitem frente à sociedade, os programas de incentivo do governo como o Prouni (Programa Universidade para Todos) e FIES (Fundo de Financiamento do Ensino Superior) foram citados por doze entrevistados como importantes instrumentos de ingresso em um curso superior.

Revista Eletrônica do Alto Vale do Itajaí - REAVI, v.08, n 13, p. 001-012, dez 2019 ISSN: 23164190, DOI 10.5965/2316419008112019001 
Vou fazer o FIES pelo fato de não ter o dinheiro para pagar a faculdade agora. E também eu acho que facilita muito a vida da gente. Muito mais (...) (E23)

Sim, por exemplo, a prova do ENEM eu já fiz, e a inscrição no Prouni também. Agora, o FIES é a segunda alternativa, mas o governo está cortando gasto com o FIES, a gente tem que conseguir passar no Prouni. (E22)

Para os estudantes, o PROUNI é percebido como uma garantia de acesso e oportunidade de estudo a quem tem dificuldade de entrar no universo acadêmico, consequentemente, contribuindo para a inclusão social. Em outra perspectiva, o FIES seria uma segunda opção, especialmente em função das novas diretrizes econômicas do governo que tem promovido cortes no financiamento da educação.

Quando questionados sobre a importância da infraestrutura da IES, os entrevistados afirmaram que conhecer os espaços físicos de uma IES traz mais segurança e certeza sobre a decisão que a ser tomada.

Como parte da infraestrutura foram citadas as instalações tecnológicas, o fácil acesso e a qualidade do imóvel. Estes aspectos corroboram o afirmado por Martins et al. (2009), quando destacam a infraestrutura como importante reflexo para o valor de uma marca, especialmente, no caso da prestação de um serviço, à medida que esta pode deixá-lo mais tangível.

$\mathrm{Na}$ dimensão preço, identificou-se que os entrevistados têm dúvidas sobre as marcas que possuem maior ou menor preço, conforme evidenciado nos relatos de E2 e E23.

Não sei, não 'tô' por dentro! (E2)

Olha, eu nunca pesquisei sobre isso, não! (E23)

Contudo, apesar de não identificarem a priori os preços das IES, este foi descrito como um diferencial na escolha, na condição de não aprovação em uma IES pública.

Eu acho que sim, pelo fato da minha família não ter condição de pagar uma faculdade boa assim, eu acho que sim (E21).

Eu sei que eu não vou ter condições de pagar uma faculdade muito cara agora. Então, eu acho que a que for mais barata vai me favorecer (E23).

Ressalta-se que o preço é uma das dimensões mais importantes para a gestão de uma organização dentro de seu nicho de mercado. A imagem articulada ao preço é um dos fatores fundamentais nos procedimentos de escolha de um serviço ou produto (BOLTON, WARLOP, ALBA, 2003; ZEITHAML, 1988).

Contudo, apesar de o preço ter sido exaltado como um fator importante na decisão por uma IES, esse revela dois sentidos. No primeiro, os preços mais baixos favorecem o acesso; no segundo, esses são associados à redução na qualidade do serviço ofertado.

É difícil, mas, às vezes, o preço influencia muito, porque bons professores não são baratos. (E1)

Em partes, sim; em partes não. Uma faculdade que preze não vai cobrar muito barato. Se for um professor bom, que tá ali disposto a ajudá-lo, ele não vai cobrar barato, ele vai cobrar caro. (E3)

Revista Eletrônica do Alto Vale do Itajaí - REAVI, v.08, n 13, p. 001-012, dez 2019 ISSN: 23164190, DOI 10.5965/2316419008112019001 


\section{REAVI}

Sousa e Sousa (2009) também encontraram essa relação dicotômica entre preço e qualidade. Segundo os autores, o preço, apesar de ser uma via de acesso, também pode representar um fator de exclusão para a escolha.

\section{Considerações Finais}

A decisão acerca de qual IES escolher pode sofrer influência de uma série de variáveis. Este estudo objetivou analisar como os estudantes estabelecem suas decisões para a escolha de uma instituição de ensino superior, na perspectiva de alunos em curso do terceiro ano do Ensino Médio.

Identificou-se forte influência dos grupos de referência para a escolha de uma IES. Essa influência pode ser percebida, especialmente, na figura dos pais, irmãos e amigos próximos. Neste aspecto, parece haver a formação de um consenso sobre a 'qualidade da instituição', construído a partir de comentários que passam de pessoas a pessoas, ou, conforme Sousa e Sousa (2009), os comentários boca a boca.

No mesmo sentido, forma-se uma percepção à cerca da qualidade que está vinculada ao preço cobrado pelos serviços educacionais e, da mesma forma que preços mais baixos podem ser atrativos, podem também indicar uma menor qualidade do ensino.

A respeito da qualidade do ensino parece não haver um consenso sobre o que ela implica em termos de serviço ofertado, podendo estar relacionada a infraestrutura, aos preços e ao currículo dos professores, o que, neste último caso, enseja novos trabalhos para identificar como os alunos e pais identificam a referida qualidade, e, em outra instância, o que seria um 'bom currículo' para os professores, na perspectiva desses atores.

Como implicação gerencial destaca-se a necessidade de se formar uma identidade da marca, em conformidade com a forma como a IES quer ser percebida no mercado.

Como sugestão para estudos futuros, destaca-se a importância de se entender o que representa a qualidade para pais e alunos, como também quais atributos estão relacionados a essa percepção.

\section{REFERÊNCIAS}

ALFINITO, S. Determinação de Atributos de Preferência do Consumidor na Escolha de uma Instituição de Ensino Superior no Distrito Federal. 2002. 122 f. Dissertação (Mestrado) - Curso de Economia de Empresas, Universidade Católica de Brasília, Distrito Federal, 2002.

ALÉSSIO, S. C.; DOMINGUES, M. J. C. S.; SCARPIN, J. E. Fatores determinantes na escolha por uma Instituição de Ensino Superior do Sul do Brasil. VII Seget - Simpósio de Excelência em Gestão e Tecnologia - 2010, Blumenau, v. 1, n. 1, p.1-15, jun. 2010.

ALMADA, I. W.; TONTINI, G. Critical satisfaction attributes in architectural services: comparison of clients' and architects' points of view. Production, v. 22, n. 2, p. 213- 224, 2012. BARDIN, L. Análise de conteúdo. Lisboa: Edições 70, 2006.

BOLTON, L. E.; WARLOP, L.; ALBA, J. W. Consumer perceptions of price (un) fairness. Journal of Consumer Research, v. 29, n. 4, p. 474-491, 2003.

Revista Eletrônica do Alto Vale do Itajaí - REAVI, v.08, n 13, p. 001-012, dez 2019 ISSN: 23164190, DOI 10.5965/2316419008112019001 


\section{REAVI}

BRASIL. Lei número 9.394, de 20 de dezembro de 1996. Palácio do Planalto, Presidência da República, Casa Civil, Subchefia para Assuntos Jurídicos. Disponível em: <http://www.planalto.gov.br/ccivil_03/leis/19394.htm> Acesso em: 12 jan. 2019.

CARDADOR, T. M.; PRATT, M. G. Identification management and its bases: bridging management and marketing perspectives through focus on affiliation dimensions. Journal of the Academy of Marketing Science, v. 34, n. 2, 174-184, 2006.

CERCHIARO, I. B.; MOTA, M. C. Avaliação da Qualidade do Serviço Educacional numa IES Particular: a visão do aluno de graduação sobre a qualidade percebida. XI Encontro Nacional de Marketing da ANPAD, Florianópolis, 23 a 25 de maio de 2010.

CHAUÍ, M. S. Escritos sobre a Universidade. São Paulo: Editora UNESP, 2001.

DIAS, C. L.; HORIGUELA, M. L. M.; MARCHELLI, P. S. Políticas para avaliação da qualidade do Ensino Superior no Brasil: um balanço crítico. Educação e Pesquisa, São Paulo, v. 32, n. 3, p.435-464, dez. 2006.

DUDZIAK, E. A. Competência informacional e midiática no ensino superior: Desafios e propostas para o Brasil. Universidade de São Paulo - Dt-sibi: prisma.com, São Paulo, v. 1, n. 13, p.1-19, jun. 2010.

ENGEL, J. F.; BLACKWELL, R. D.; MINIARD, P. W. Comportamento do Consumidor. Rio de Janeiro: Livros Técnicos e Científicos Editora, 2000.

FOSKETT, N.; ROBERTS, D.; MARINGE, F. Changing fee regimes and their impact on student attitudes to higher education, York: Higher Education Academy, 2006.

FRANZ, L. S.; KRAMER, M. W. The Dimensions of Decision: a conceptual and empirical investigation. In: NUTT, P. C.; WILSON, D. C. (Edit.) Handbook of -Decision Making. West Sussex: Wiley, 2010. p. 517-540.

FROEMMING, L. M. S. Encontros de Serviços em uma Instituição de Ensino Superior. 2001. 284f. Tese de Doutorado. Porto Alegre, Universidade Federal do Rio Grande do Sul, 2001.

GADOTTI, M. Educação Brasileira Contemporânea: Desafios do Ensino Básico. Produção de terceiros sobre Paulo Freire; Série Artigos, 1997.

HOFFMAN, K. D.; BATESON, J. E. G. Princípios de Marketing de Serviços: conceitos, estratégias e casos. São Paulo: Pioneira Thomson Learning, 2006.

IBDAIWI, T. K. R. Determinação das Características de Preferência dos Alunos na Escolha de uma Instituição de Ensino Superior Privada na Cidade de Santa Maria - RS. 2009. 118 f. Dissertação (Mestrado) - Curso de Mestrado em Engenharia de Produção, Centro de Tecnologia, Universidade Federal de Santa Maria, Santa Maria, 2009.

Revista Eletrônica do Alto Vale do Itajaí - REAVI, v.08, n 13, p. 001-012, dez 2019 ISSN: 23164190, DOI 10.5965/2316419008112019001 


\section{REAVI}

KAGAWA, S. K.; PERSCH, R. V. A gestão do brand equity no marketing educacional. Revista Gestão Universitária na América Latina-GUAL, v. 9, n. 3, 2016.

KOC, E. Extended review of Shakespeare, Einstein and the bottom line: the marketing of higher education by D. L. Kirp. On The Horizon, v. 14, n. 1, p. 19-21, 2006.

KOTLER, P.; FOX, K. Marketing Estratégico para Instituições Educacionais. São Paulo: Atlas, 1994.

LEVENFUS, R. S.; NUNES, M. L. T. Principais temas abordados por jovens centrados na escolha profissional. Porto Alegre: Artes Médicas, 2002.

LOVELOCK, C.; WIRTZ, J. Marketing de serviços: pessoas, tecnologia e resultados. 5. ed. São Paulo: Pearson Prentice Hall, 2006.

MAINARDES, E. W.; DOMINGUES, M. J. C. S. Atração de alunos para a graduação em Administração: estudo multicaso sobre os fatores de atratividade em cursos superiores de Joinville, SC. Revista de Economia e Administração, Joinville - Sc, v. 9, n. 1, p.77-93, mar. 2010.

MANEA, N.; PURCARU, M. The Evolution Of Educational Marketing. Annals of" Spiru Haret". Economic Series, v. 17, n. 4, p. 37-45, 2017.

MARTINS, J. M. D. et al. Marketing Educacional: uma Análise de Atributos e Posicionamento de Instituições de Ensino Superior. Revista Adm.Made, Rio de Janeiro, v. 13, n. 1, p.1-19, abr. 2009.

MASQUIETTO, C. D. et al. Marketing no ensino superior: atributos valorizados pelos discentes de curso de especialização. Revista Gestão Universitária na América Latina-GUAL, v. 10, n. 4, p. 227-251, 2017.

MAVONDO, F. T.; CHIMHANZI, J.; STEWART, J. Learning orientation and market orientation: relationship with innovation, human resource practices and performance. European Journal of Marketing, [S.1.], v. 39, n. 11/12, p. 1235-1263, 2005.

MICHAEL, S. O. American higher education system: consumerism versus professorialism. International Journal of Education Management, v. 11, n. 3, p. 117-130, 1997.

NEVES, A. B.; RAMOS, C. F. A imagem das instituições de ensino superior e a qualidade do ensino de graduação: a percepção dos acadêmicos do curso de administração, XII ENANGRAD, 2011.

PEREIRA, B. C. S.; GIL, C. Avaliando a Satisfação de Alunos de Escolas de Administração: uma nova perspectiva de gestão. Revista de Administração da Unimep, Piracicaba - Sp, v. 5, n. 1, p.1-21, abr. 2007.

Revista Eletrônica do Alto Vale do Itajaí - REAVI, v.08, n 13, p. 001-012, dez 2019 ISSN: 23164190, DOI 10.5965/2316419008112019001 


\section{REAVI}

PALÁCIO, A. B.; MENESES, G. D.; PÉREZ, P. J. P. The configuration of the university image and its relationship with the satisfaction of students. Journal of Educational Administration, [S.1.], v. 40, n. 5, p. 486-505, 2002.

REICHELT, V. P. A Gestão do Brand Equity no Marketing Educacional. Revista Gestão Universitária da América Latina, v. 9, p. 269-291, 2016.

ROWE, D. E.O.; BASTOS, A. V. B. Comprometimento organizacional e desempenho acadêmico: um estudo com docentes do ensino superior brasileiro. In: XXX Encontro da Associação Nacional de Pós-Graduação e Pesquisa em Administração, São Paulo, 2009.

SAKATA, M. C. G. et al. Políticas de avaliação da educação e quase mercado no Brasil. Educação Social. Campinas, v. 24, n. 84, p. 873-895, set./ 2003.

SIQUEIRA, R. P.; CARVALHO, J. L. F. Qualidade do Serviço Educacional Prestado por Escolas de Administração: Confronto Entre uma Universidade Pública e uma Faculdade Privada. Anais do XXX Enanpad, Salvador/BA, set./2006.

SOLOMON, M. R. O comportamento do consumidor: comprando, possuindo e sendo. 5. ed. Porto Alegre: Bookman, 2002.

SOUSA, A.; NETO, A.; FONTENELE, R.; Determinantes da intenção da escolha do ensino superior privado. Revista Eletrônica de Ciência administrativa (RECADM), Campo Largo, v.12, n.3, p. 366-377, Set-Dez/ 2013.

SOUSA, E.; SOUSA, C.; Determinantes da escolha de instituições de ensino superior: uma visão dos alunos do ensino médio em Belo Horizonte. Revista Educação \& Tecnologia, Belo Horizonte/MG, v.14, n.1, p. 28-35, jan./abr. 2009.

SÉCCA, R. X.; LEAL, R. M. Análise do setor de ensino superior privado no Brasil. Educação: BNDES Setorial, São Paulo, v. 1, n. 30, p.103-156, ago. 2009.

SANTOS, M. J. M. et al. Heurísticas no Consumo de Serviços De Educação Superior Revista Brasileira de Marketing, v. 9, n. 1, p. 40-63, 2010.

SEEMAN, E. D.; O'HARA, M. Customer relationship management in higher education using information systems to improve the student-school relationship. Campus-Wide Information Systems, v. 23, n. 1, p. 24-34, 2006.

TARI, J. J. An EFQM model self-assessment exercise at a Spanish university. Journal of Educational Administration, [S.1.], v. 44, n. 2, p. 170-188, 2006.

VIGGIANO, E.; MATTOS, C. O desempenho de estudantes no ENEM 2010 em diferentes regiões brasileiras. Revista Brasileira de Estudos Pedagógicos, Brasília, v. 94, n. 237, p. 417-438, mai/ago. 2013.

Revista Eletrônica do Alto Vale do Itajaí - REAVI, v.08, n 13, p. 001-012, dez 2019 ISSN: 23164190, DOI 10.5965/2316419008112019001 


\section{REAVI}

ZEITHAML, V. L. Consumer perceptions of price, quality and value: a means-end model and synthesis of evidence. Journal of Marketing, v. 52, n. 3, p.2-22, 1988.

ZEITHAML, V.; BITNER, M. J. Services Marketing: integrating customer across the firm. New York, McGraw-Hill, 2000.

Revista Eletrônica do Alto Vale do Itajaí - REAVI, v.08, n 13, p. 001-012, dez 2019 ISSN: 23164190, DOI 10.5965/2316419008112019001 